\title{
Design of Aerial Platform Wireless Networks for Surveillance and Monitoring Applications
}

\author{
Fahad Alraddady \\ Computer Engineering Department, College of Computers and Information Technology, Taif University, Saudi Arabia.
}

\begin{abstract}
In this paper, the Aerial Platform (AP) Wireless Sensor Network (WSN) is modelled and demonstrated for surveillance applications such as traffic monitoring and management. The system geometrical model and coverage parameters of AP such as coverage diameter, beamwidth of beam patter, and coverage area per AP are investigated where the AP network design equations are deduced for several WSN scenarios which include the coverage of rural areas linked by highways and urban cities as well. Also, the sensor data rate and density in the coverage area are related to the AP coverage parameters and a set of design curves are developed which determines the coverage diameter required per AP at a specific sensor data rate and uplink capacity for the whole AP covered area. The sensors are assumed of two kinds which are low-speed sensors for monitoring slowly varying physical quantities and high-speed sensors such as those used in video monitoring.
\end{abstract}

Keywords: Aerial platforms, wireless sensor network, traffic monitoring, surveillance systems.

\section{INTRODUCTION}

Aerial Platforms (AP) have gained attention recently as it provides several capabilities for many applications including monitoring, surveillance, communication, traffic control and many others [1-9]. AP may be in the form of balloon filled with helium or hydrogen which is called lighter-than air platform or may be in the form of unmanned-aerial vehicle which remotely controlled during flight missions [10-11]. One of the most important applications of AP is the wireless communication networks that can be established in very short time without needing a huge and costly infrastructure. In a few minutes, a basestation in the form of an AP can be launched to provide very wide wireless coverage and sometimes it can extend to one-thousand-kilometer diameter if maintained at $20 \mathrm{~km}$ high in the stratosphere [12]. This huge coverage distance could provide coverage to a whole country or sometimes many neighbored countries. On the other hand, an AP may be launched at lower altitude for rapid and short missions including drones applications for imagery and monitoring. For surveillance applications, the AP station may act as a wide area sink for the monitoring sensors and cameras or a wide area aerial sensor or camera. The first topology suits the coverage of heavy and moderate traffic regions while the second one suits the rural and very low traffic areas such as deserts and sea regions. Therefore, in this paper, the first topology will be demonstrated and investigated where the data from ground sensors are sent directly or through local sink station to the wide area AP sink. This network architecture acts as standalone network which uses wireless connection over free or dedicated communications frequencies. The advantages of using AP in this network includes improved system security, better coverage and communications performance, wide area coverage, capability to connect and reach uncovered areas such as deserts, mountains, sea, or isolated valleys which will be very important for surveillance and rescue operations for lost trucks or people [13-15]. The AP network installation should start with the geometrical analysis of the covered areas and highways which will be described in the next sections. The paper is arranged as follows: Section II demonstrates the geometrical specifications and aspects of AP wireless sensor network (WSN). Section III provides a design for AP WSN for rural areas while Section IV design an urban city AP WSN. Section V demonstrates the AP WSN with variable senor data rates and finally Section VI concludes the paper.

\section{GEOMETRY OF THE AP WirELESS SENSOR NETWORK}

To design AP system for WSN applications, we first provide geometrical analysis which defines the coverage area and its geometrical parameters, and the required number of APs needed for the network installation. Consider anAPthat is located at $h \mathrm{~km}$ above ground as shown in Fig. (1). The coverage area can be determined if we find the diameter $D_{c}$ of resulted from a beam from the AP and the coverage beamwidth is the contour of the $3 \mathrm{dBreceived} \mathrm{power.}$ 


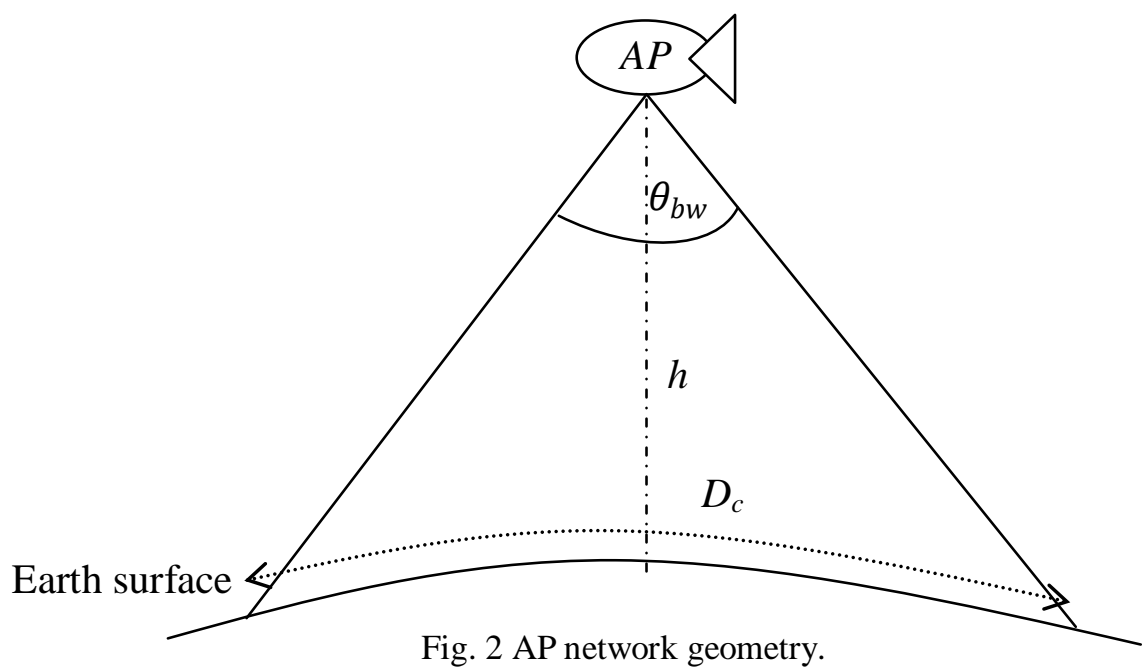

If the radio coverage beamwidth is $\theta_{b w}$, then for APs at very low altitudes, the coverage diameter is defined as follows:

$$
D_{c} \approx 2 h \tan \left(\frac{\theta_{b w}}{2}\right)
$$

Eq. (1) can be used for small AP stations such as air drones or for APs covering small areas where the slant range of the coverage area $S_{h}$ is much smaller than the tangential range.

The analysis and variation of the coverage distance is shown in Figs. (2) and (3) for very low and very high AP altitudes. The coverage diameter will increase when we increase the AP height or beamwidth. For large area coverage we may use wider beams at high altitudes while for achieving wider area coverage at low altitudes, we should use very wide beams.

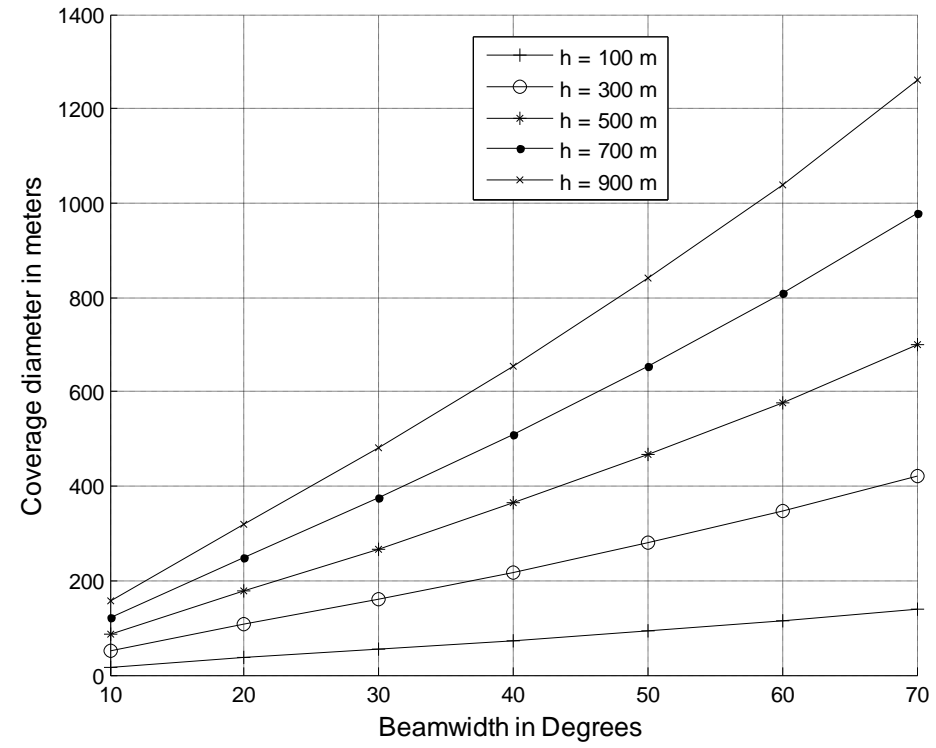

Fig. 2 Coverage diameter of AP versus beamwidth at different low AP altitudes.

For AP network installation, we need to define required of AP stations needed to cover the intended area which may be a rural area with very long highways or an urban area such as city and dense populated regions and this topic will be discussed in the next section. 


\title{
International Journal of Advanced Research in Computer and Communication Engineering
}

\author{
Vol. 9, Issue 9, September 2020
}

DOI 10.17148/IJARCCE.2020.9911

\section{III.AP WSN fOR RURAL AREAS}

The coverage of rural areas is characterized by large coverage diameter and sparse sensor locations which are localized on the roadsides and crossings. The sensor density is very low and may be few tens per square kilometer. Also, the area includes desert regions, mountains, hilly regions, and sear shores.

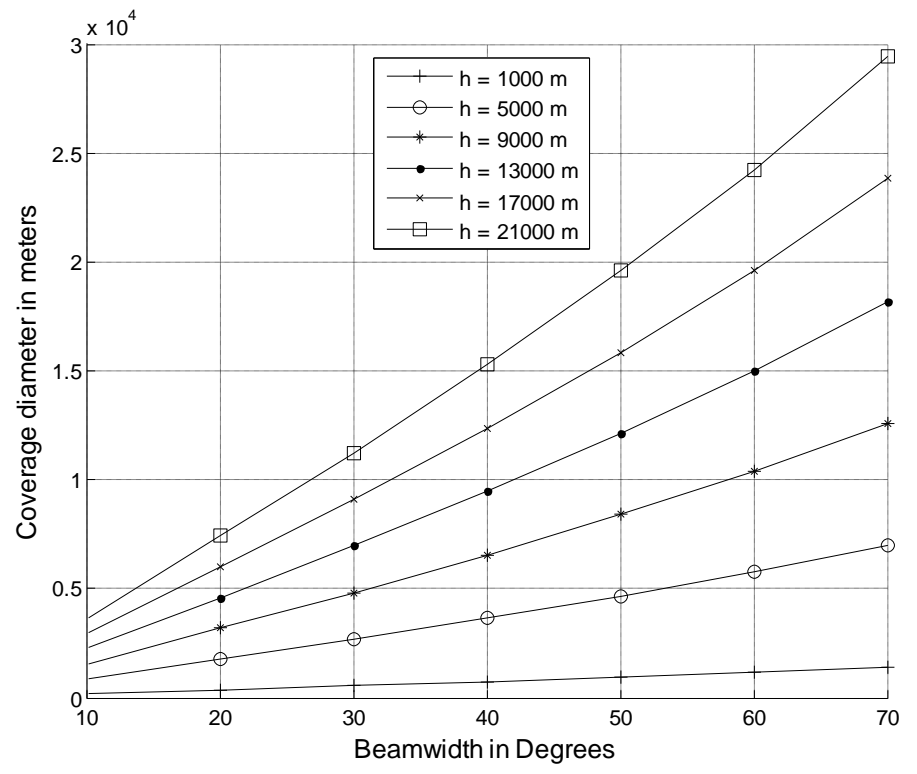

Fig. 3 Coverage diameter of AP versus beamwidth at different high AP altitudes.

A single AP can serve few hundreds of kilometres of coverage diameters, but this will require high transmitting power from sensors on the ground surface resulting in limited lifetime for these sensors especially in the desert and regions of limited power sources. Therefore, we assume few tenses of kilometres as a coverage diameter which requires several AP stations to cover very long highways.

According to the lengths of the highway segments for each AP, we can determine the number of APs needed to install this network. Assuming that the $m^{\text {th }} \mathrm{AP}$ has a coverage diameter of $D_{c m}$ and the total highway length covered by the AP network is $L$, then we can divide this length into a number of segments in two approaches; the first is equal highway segment lengths or equal coverage diameters coverage. In the first approach, the AP stations may have different coverage diameters due to the curls that may exist in the highway over the whole covered area while the second approach, we maintain the equal coverage diameters of the different APs regardless the actual lengths of the road segments. For the two cases $L$ is related to the AP coverage diameters by the following relation:

$$
L \leq \sum_{m=1}^{M} D_{c m}
$$

We assume here for simplicity that the sensors have equal density over the covered areas of each AP and the road is almost straight or has uniform curvatures therefore the two approaches will have the same number of APs needed to build the network. Therefore, $L$ is related to the coverage diameters by the following equation:

$$
L=M D_{c}
$$

And the number of AP stations is thus given by:

$$
M=\left\lceil\frac{L}{D_{c}}\right\rceil
$$

Where $\lceil ~\rceil$ is an operator that gives the nearest highest integer number.

The number of AP stations can be optimized if we include the platforms heights in the system design provided that the legal heights are utilized and the sensor transmitting power margins are not exceeded. For example, longer road segments with low density of traffic sensors are covered by higher altitude AP while segments with higher traffic sensor density can be covered by low altitude platforms. This will reduce the number of AP stations required in the system. 


\section{IV.APNETWORK FOR CITIES AND URBAN AREAS}

The coverage scenario in the case of cities and populated urban areas will differ from the previous case where the number of cross-roads increases, and the streets almost have two-dimension distribution as shown in Fig. (4). At some places multilevel streets exist which include bridges and underground. This type of traffic is characterized by the high senor density (or increased number of sensors per square kilometer) and consequently smaller coverage diameters should be provided through smaller beamwidths or low altitude platforms.

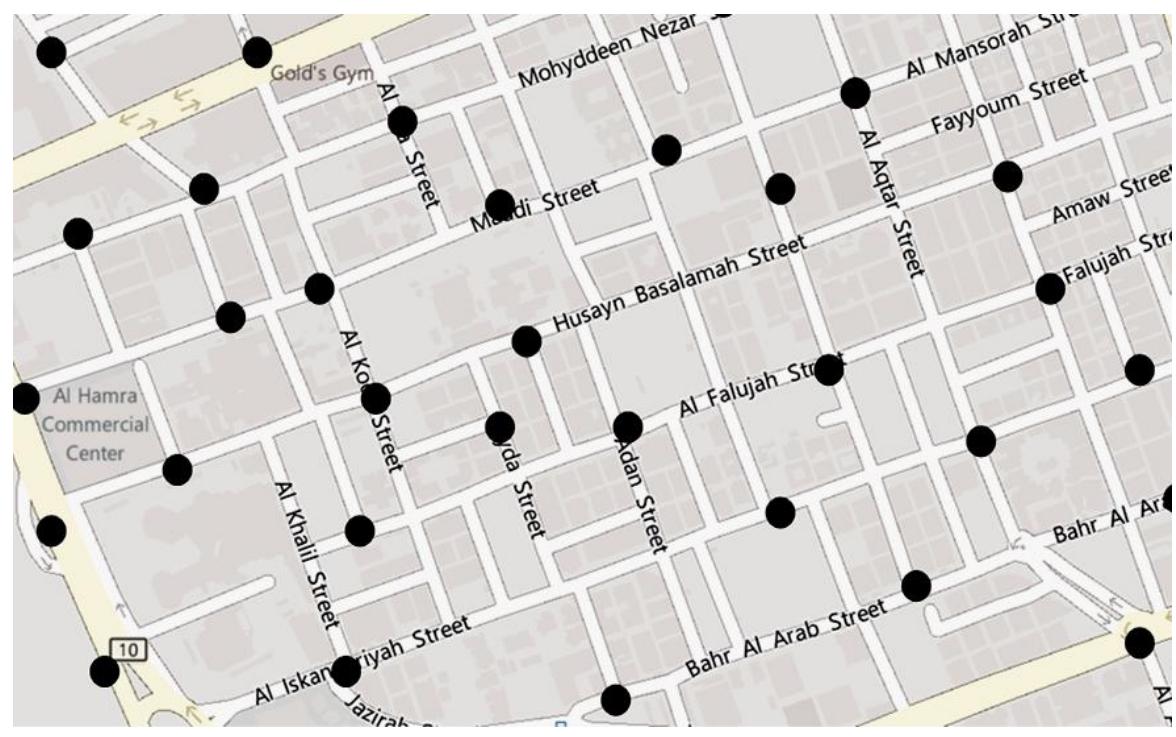

Fig. 4 City streets structures and multidimensional sensor distribution

The number of the sensors per unit area is calculated by counting the number of sensors in the two-dimensional road system. If the unit area contains $I \times J$ street segments where the $i^{\text {th }}$ and $j^{\text {th }}$ segments have $u_{i}$ and $k_{j}$ sensor densities respectively, then the sensors density is given by:

$$
v=\sum_{i=1}^{I} \sum_{j=1}^{J} u_{i} k_{j}
$$

Therefore, if the $m^{\text {th }} \mathrm{AP}$ has a communication sensor capacity $C_{m}$, then the covered area of the $m^{\text {th }} \mathrm{AP}$ is given by

$$
A_{h m}=\frac{C_{m}}{v}
$$

And the number of AP stations required for covering the whole city will be

$$
M=\frac{\text { Total City Area }}{A_{h m}}
$$

Or

$$
M=v \frac{\text { Total City Area }}{C_{m}}
$$

So, increasing the AP sensor capacity will reduce the required number of the AP stations which means that the AP can serve large number of sensors and can cover wider area. This conclusion is true if the sensor density over the city area is kept constant.

\section{APNETWORK DESIGN FOR VARIABLE SENSOR DATA RATES}

The design of AP wireless sensor network provides the AP coverage area or diameter for specific system parameters such as AP altitude, communication capacity or the number of communications channels for sensors, the available sensor technology which defines the transmitted sensor power, etc. The sensors data rate varies according to the type of transmitted information. The measurements may include low data rates as well as high data rates especially video 
transmission from high resolution cameras. Therefore, in general, we can classify the sensor types into two main categories namely, low, and high data rate sensors. Assume that in the AP coverage area, the number of low data rate sensors is $n_{L}$ and that for high data rate sensors is $n_{H}$. The corresponding sensor data rates are $r_{L}$ and $r_{H}$ respectively. So, the total uplink data rate (excluding headers or other control signals) will be:

$$
R_{u}=\sum_{i=1}^{n_{L}} r_{L i}+\sum_{j=1}^{n_{H}} r_{H j}
$$

According to the number and type of sensors, we can determine the proper coverage diameter at a specific AP altitude and sensor density. Assume that the density of the low data rate sensors is $v_{L}$ and that for the high data rate sensors is $v_{H}$ and all the sensors are uniformly distributed over the AP coverage area, then the bite rates per unit area is given by:

$$
R_{u L \mid A}=r_{L} v_{L}
$$

And

$$
R_{u H \mid A}=r_{H} v_{H}
$$

And the total sensor uplink data rate per unit area is given by

$$
R_{u \mid A}=R_{u L \mid A}+R_{u H \mid A}
$$

Or

$$
R_{u \mid A}=r_{L} v_{L}+r_{H} v_{H}
$$

Therefore, the total uplink data rate will be

$$
R_{u}=\pi\left(\frac{D_{c}}{2}\right)^{2}\left(r_{L} v_{L}+r_{H} v_{H}\right)
$$

And the coverage diameter $D_{c}$ is thus given by:

$$
D_{c}=2 \sqrt{\frac{R_{u}}{\pi\left(r_{L} v_{L}+r_{H} v_{H}\right)}}
$$

And generally, for any number of different rates sensors, $K$, the coverage radius will be given by:

$$
D_{c}=2 \sqrt{\frac{R_{u}}{\pi \sum_{k=1}^{K} r_{k} v_{k}}}
$$

This is the main target equation for the design of AP network where the sensor densities and data rates as well as the uplink overall capacity of the system determines the coverage diameter. The variation of the system coverage area with the sensor density (considering the mentioned two practical sensor types) at different uplink capacity is shown in Figs. (5) to (10) where these figures may be used as design curves based on the last coverage diameter equation. Generally, for low speed senor data rates, the coverage diameter of the AP decreases as the sensor density increases at a constant uplink capacity. Also increasing the high-speed senor density reduces the coverage area per AP and results in a requirement for larger number of APs to cover the same area.

\section{VI.CONCLUSION}

In this paper, the AP has been used in wireless senor network applications such as traffic management and surveillance. The AP network designs have been demonstrated starting by geometrical investigations and modelling where the coverage aspects of AP are deduced as well as sensor data rates at different uplink capacities. Several designs have been investigated including rural and urban regions where the number of APs forming a network has been deduced for rural highway regions as well as urban cities. It is found that the deduced number of APs required to build a network for wide area coverage is related to the sensor density, AP height, sensor data rates and the uplink capacity per AP. In addition, a set of design curves have been produced to determine the AP coverage diameter required at specific sensor data rate and uplink AP capacity which helps in designed AP WSN. 


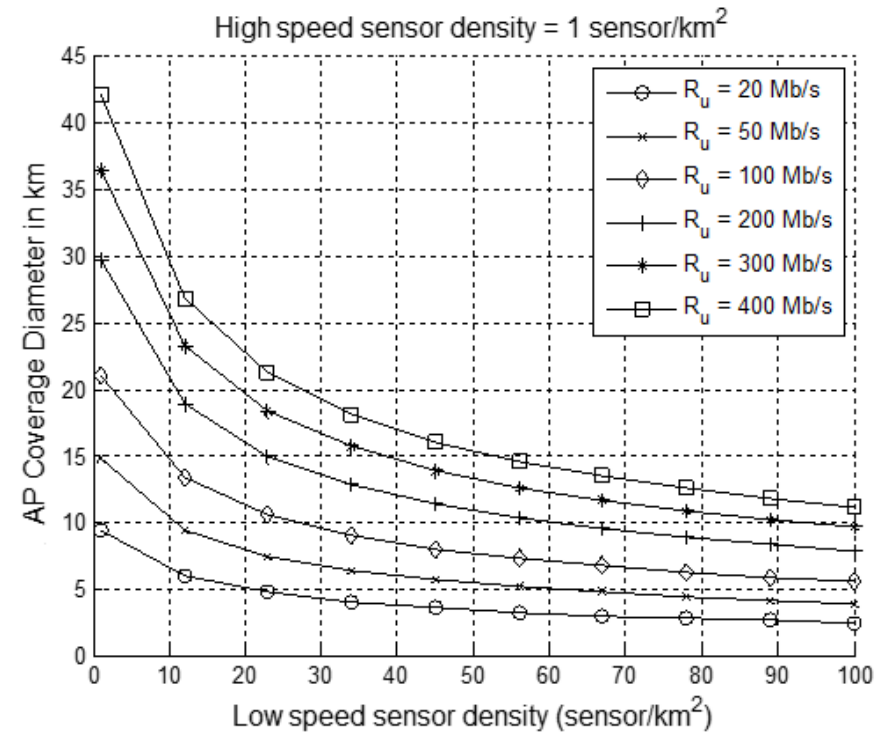

Fig. 5AP coverage diameter variation with the low speed sensor density at high speed sensor density of 1 sensor $/ \mathrm{km}^{2}$ and different uplink capacities.

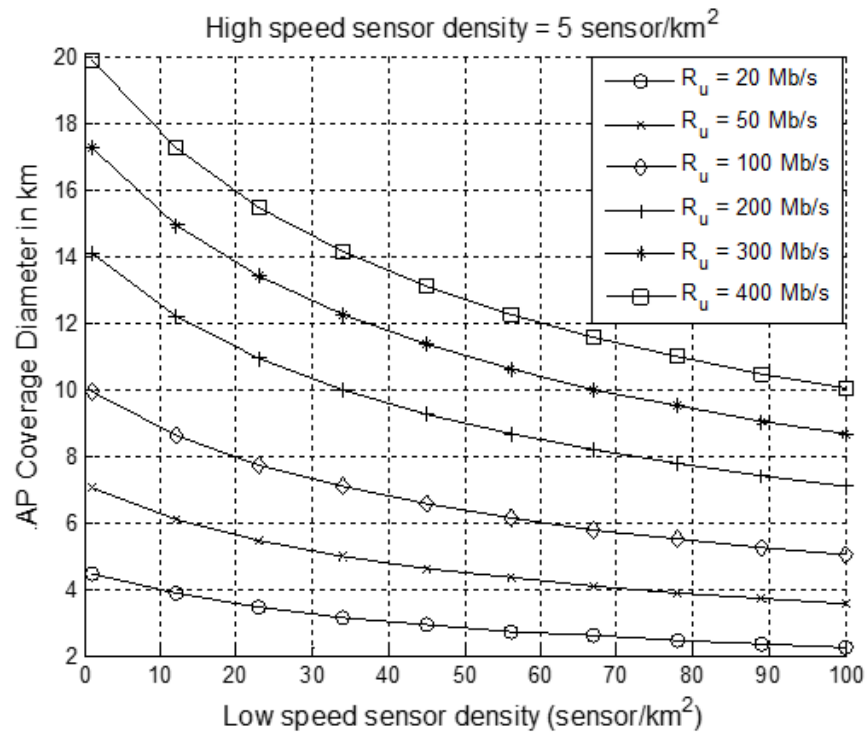

Fig. 6AP coverage diameter variation with the low speed sensor density at high speed sensor density of 5 sensor $/ \mathrm{km}^{2}$ and different uplink capacities. 


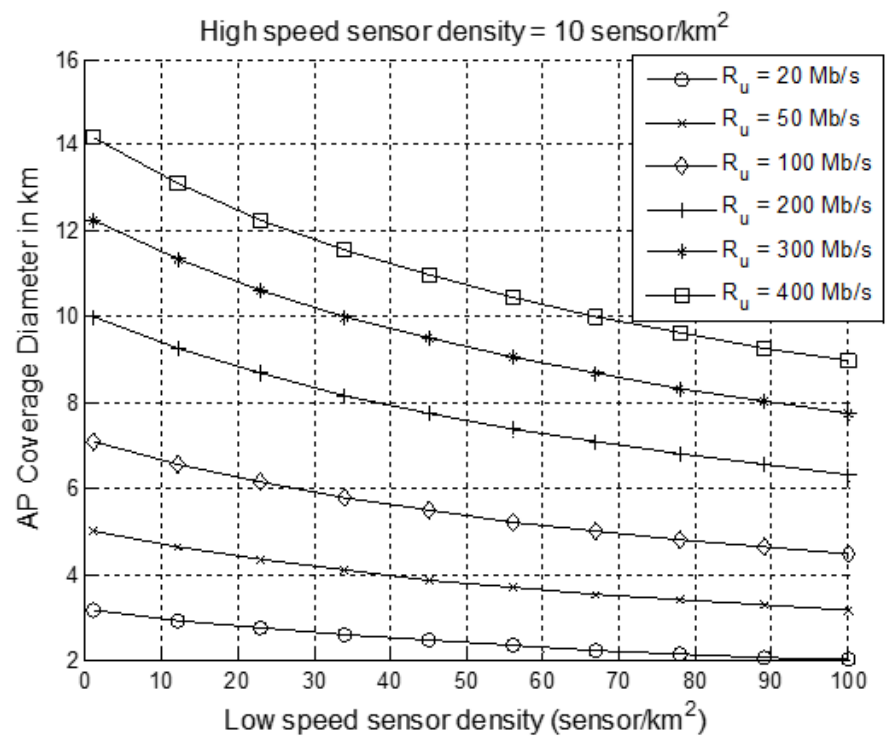

Fig. 7AP coverage diameter variation with the low speed sensor density at high speed sensor density of $10 \mathrm{sensor} / \mathrm{km}^{2}$ and different uplink capacities.

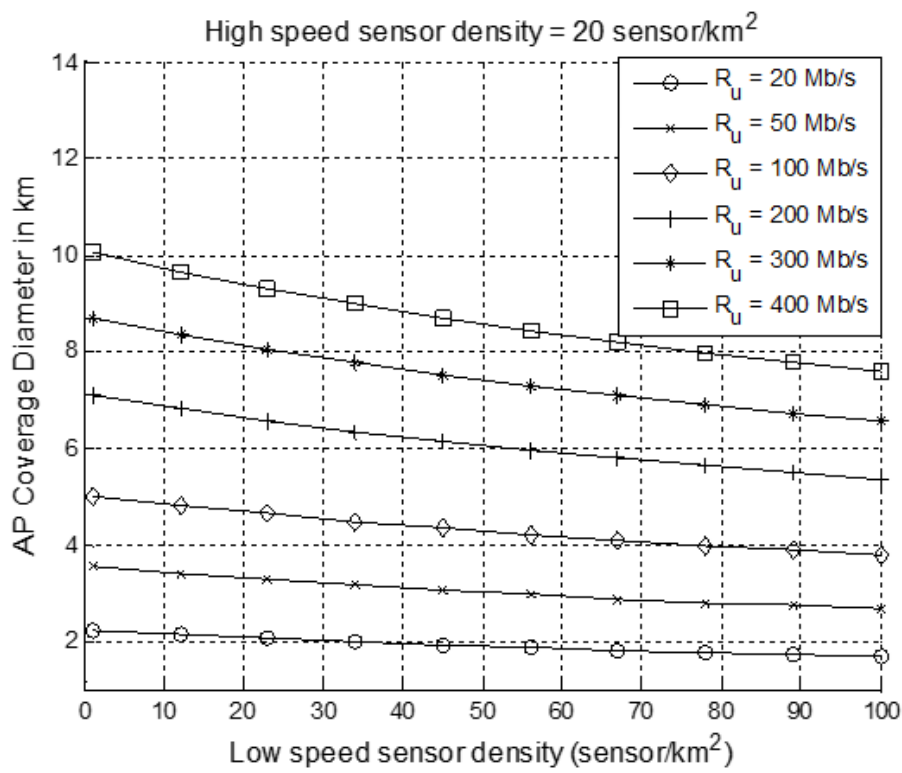

Fig. 8AP coverage diameter variation with the low speed sensor density at high speed sensor density of $20 \mathrm{sensor} / \mathrm{km}^{2}$ and different uplink capacities. 


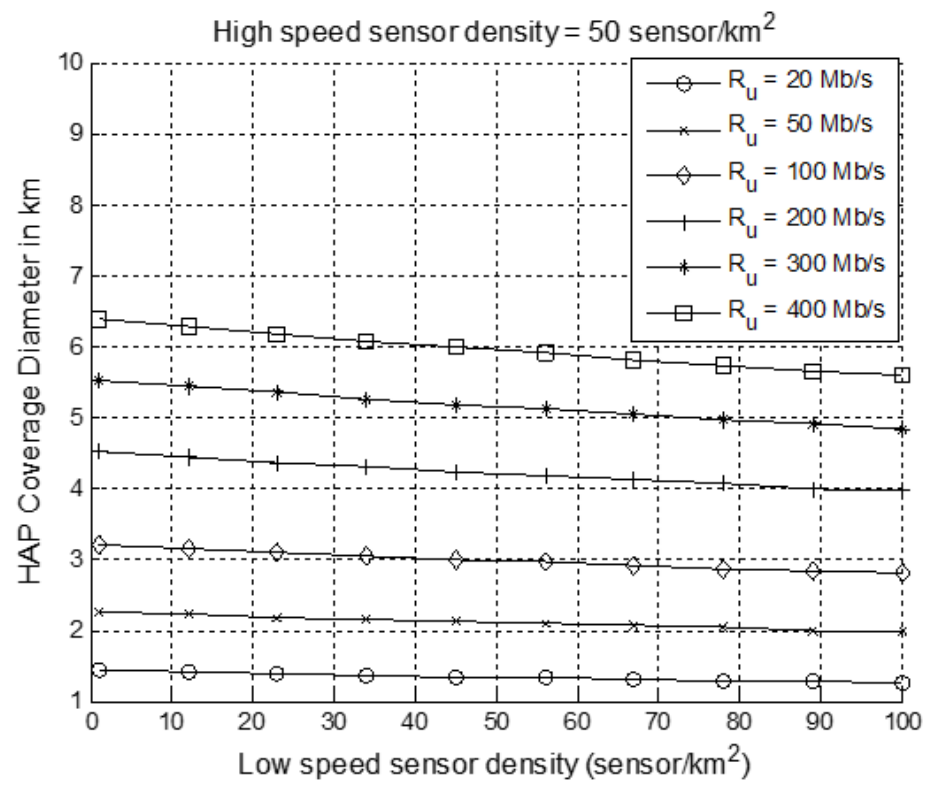

Fig. 9AP coverage diameter variation with the low speed sensor density at high speed sensor density of 50 sensor $/ \mathrm{km}^{2}$ and different uplink capacities.

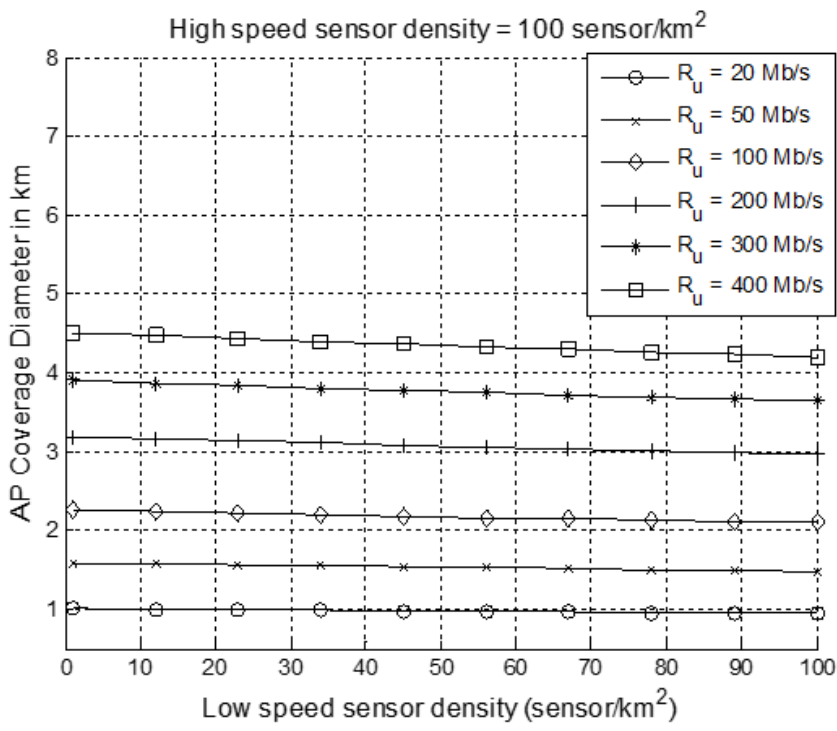

Fig. 10 AP coverage diameter variation with the low speed sensor density at high speed sensor density of 100 sensor $/ \mathrm{km}^{2}$ and different uplink capacities. 


\title{
International Journal of Advanced Research in Computer and Communication Engineering
}

\author{
Vol. 9, Issue 9, September 2020
}

DOI 10.17148/IJARCCE.2020.9911

\section{REFERENCES}

[1] Breitling Orbiter, see https://en.wikipedia.org/wiki/Breitling_Orbiter

[2] Lindstrand Balloons Ltd., see http://www.lindstrand.com

[3] Project Loon, see https://x.company/loon/

[4] AeroVironment, see http://www.avinc.com/

[5] Global Hawk, see:http://www.northropgrumman.com/Capabilities/GlobalHawk/Pages/default.aspx

[6] Predator, see http://www.gat.com/asi/aero.html

[7] COMETS Project http://www.comets-uavs.org/

[8] MARVIN Helicopter http://pdv.cs.tu-berlin.de/MARVIN/tubrob97.html

[9] CAMCOPTER http://www.schiebel.net/pages/html_version.html

[10] Unmanned Aerial Vehicles project at EidgenossischeTechnische Hochschule (ETH) Zurich. http://www.uav.ethz.ch

[11] Eisenbeiss, H., “A Mini Unmanned Aerial Vehicle (UAV): System Overview and Image Acquisiton” International Workshop on Processing and Visualization using High-Resolution Imagery, November 2004, Thailand.

[12] A. A. Zaidi, B. Kulcsár and H. Wymeersch, "Back-Pressure Traffic Signal Control With Fixed and Adaptive Routing for Urban Vehicular Networks," in IEEE Transactions on Intelligent Transportation Systems, vol. 17, no. 8, pp. 2134-2143, Aug. 2016.

[13] Mike Smith, "Traffic signal control and route choice, A new assignment and control model which designs signal timings", Transportation Research Part C: Emerging Technologies, Volume 58, Part C, September 2015, Pages 451-473.

[14] Jens Leitloff, Dominik Rosenbaum, Franz Kurz, Oliver Meynberg and Peter Reinartz, "An Operational System for Estimating Road Traffic Information from Aerial Images", Remote Sensing, vol. 11, pp. 11315-11341, 2014, doi:10.3390/rs61111315.

[15] Sebastien Razakarivony and Frederic Jurie, "Vehicle detection in aerial Imagery: A small target detection benchmark", Journal of Visual Communication and Image Representation, vol. 34, issue C, pp. 187-203, January 2016. 